\title{
Ingredientes usados na indústria de chocolates
}

\author{
Marissol Richter, Suzana Caetano da Silva Lannes*
}

Departamento de Tecnologia Bioquímico-Farmacêutica, Faculdade de Ciências Farmacêuticas, Universidade de São Paulo

*Correspondência

S. C. S. Lannes

Departamento de Tecnologia

Bioquímico-Farmacêutica

Faculdade de Ciências Farmacêuticas

Universidade de São Paulo

Av. Dr. Lineu Prestes, 580 - Bloco 16

05508-900 - São Paulo - SP, Brasil

E-mail: scslan@usp.br
Chocolate é um produto comumente consumido, sendo que sua produção tem aumentado nos últimos anos. Produzir chocolates requer um entendimento do consumidor. Os tipos preferidos de chocolate variam em cada país. Os diferentes sabores e usos para o chocolate refletem a história da indústria dos diferentes lugares. O sabor do chocolate é parcialmente determinado pela química do produto. O sabor depende da liberação dos compostos aromáticos, enquanto que a textura é uma função da maneira como o material se funde e quebra na boca. Muitos chocolates disponíveis no mercado são elaborados com ingredientes similares, porém apresentam diferentes sabores. Alguns produtores têm aromas específicos, sendo que existem trocas freqüentes nestes devido a variações no processo, acidez e temperatura, ocasionando variações de aroma e sabor no produto final. Por esta razão, as condições de produção do chocolate são mantidas freqüentemente em segredo. Os ingredientes utilizados na produção de chocolates e de seus produtos têm importante papel na aceitação pelo consumidor e na apresentação do produto.
Unitermos

- Chocolate

- Bombom

- Ingredientes/indústria alimentícia

- Indústria alimentícia

\section{INTRODUÇÃO}

Chocolate é o produto obtido a partir da mistura de derivados de cacau (Theobroma cacao L.), massa (ou pasta ou liquor) de cacau, cacau em pó e ou manteiga de cacau, com outros ingredientes, contendo, no mínimo, $25 \%$ (g/100 g) de sólidos totais de cacau. O produto pode apresentar recheio, cobertura, formato e consistência variados. Chocolate branco é o produto obtido a partir da mistura de manteiga de cacau com outros ingredientes, contendo, no mínimo, 20\% (g/100 g) de sólidos totais de manteiga de cacau. $\mathrm{O}$ produto pode apresentar recheio, cobertura, formato e consistência variados (Brasil, 2005).
A confecção do chocolate foi, durante um século, uma indústria tradicional administrada por artesãos que desenvolveram métodos individuais de trabalho e sabores particulares para os seus produtos. Com a demanda por custos mais baixos, a manufatura industrial foi sendo cada vez mais mecanizada, somando-se a isso o progressivo avanço da ciência e da tecnologia para controle das plantas de produção e para a melhoria da eficiência industrial (Bonzas, Brown, 1999).

A composição precisa do chocolate varia em todo o mundo devido à diferença de gostos e legislação, que se preocupa com as porcentagens de cacau e sólidos do leite adicionais, quantidade e tipos de gorduras vegetais permitidas. 
As gorduras encontradas no chocolate incluem a manteiga de cacau, a gordura do leite e gordura vegetal (Martin, 1994)

A gordura do chocolate, derivada do cacau, é constituída por dois ácidos graxos saturados, o ácido palmítico e o esteárico, e o ácido oléico monoinsaturado, em adição de uma pequena quantia (menos do que $5 \%$ ) de outros ácidos graxos. Embora se acredite que o consumo de gorduras saturadas aumenta o nível plasmático de colesterol, o consumo regular de manteiga de cacau e chocolate vem negando este aumento (Wang et al., 2000). A maior parte das pesquisas vem demonstrando que isto se deve, provavelmente, às concentrações relativamente altas de ácido esteárico, que tem mostrado um efeito neutro sobre o metabolismo do colesterol, e ao ácido oléico, conhecido pelos seus efeitos na redução plasmática do colesterol médio. Além dos carboidratos simples e da gordura presentes no chocolate, o componente do cacau é rico em inúmeros minerais essenciais, como magnésio, cobre, potássio e manganês (Hammerstone et al., 1999).

Para manipulação adequada o chocolate deve estar derretido. Isso se faz necessário porque a manteiga de cacau (presente no chocolate) possui formas cristalinas distintas (polimorfismo), sendo que cada uma delas possui pontos de fusão diferentes (Bouzas, Brown, 1999). Das formas cristalinas, a mais estável às oscilações da temperatura ambiente é a forma $\beta$ (VI), enquanto as demais sofrem transições e se fundem. Para se obter um produto à base de manteiga de cacau com boas características de qualidade (textura, cor e brilho), após o completo derretimento sem nenhuma forma cristalina presente (temperatura entre $45^{\circ} \mathrm{C}$ e $50^{\circ} \mathrm{C}$ ), inicia-se o processo de pré-cristalização (temperagem) com resfriamento lento e gradual, com movimentação constante, induzindo a formação dos cristais $\beta$ (VI) estáveis de maneira homogênea (McGauley, Marangoni, 2002; Schenk, Peschar, 2004).

A pré-cristalização ocorre em 2 a $4 \%$ da manteiga de cacau, causando aumento da viscosidade. No final da temperagem, o chocolate apresenta viscosidade duas vezes maior quando comparada com o chocolate derretido inicialmente. A temperatura adequada para o final de temperagem varia de acordo com o tipo de chocolate, sendo por volta de $28^{\circ} \mathrm{C}, 29^{\circ} \mathrm{C}$ e $31^{\circ} \mathrm{C}$, para os chocolates branco, ao leite e meio amargo, respectivamente (Schenk, Peschar, 2004). No resfriamento (após a modelagem), acelera-se a multiplicação dos cristais $\beta$ (VI), formando uma rede cristalina compacta e estável. Entre a temperatura de resfriamento (túnel ou geladeira, de 8 a $10^{\circ} \mathrm{C}$ ) e o ambiente de trabalho $\left(20\right.$ a $\left.22{ }^{\circ} \mathrm{C}\right)$ não deve haver uma diferença superior a $10^{\circ} \mathrm{C}$, para evitar a condensação de umidade sobre o produto (Stapley et al.,1999; Mcgauley,
Marangoni, 2002). Uma temperagem correta garante que a manteiga de cacau irá se contrair no molde e o chocolate saíra facilmente (Martin, 1994).

O chocolate vem ganhando cada vez mais espaço na mídia, não só pelas suas apreciadas propriedades sensoriais, mas também pelos benefícios potenciais à saúde. Uma análise mais profunda, baseada em pesquisas científicas, sugere que, realmente, alguns chocolates podem ter o potencial de contribuir beneficamente para a saúde quando consumidos com moderação. Entretanto, é ainda prematuro dizer que esta evidência promissora é conclusiva e cuidados devem ser tomados para interpretar e representar devidamente esta informação (Schmitz, 2001).

Com o passar dos anos, o cacau foi sendo reconhecido pelo seu conteúdo de fitoquímicos, especialmente pela metil-xantina e pela teobromina, substâncias com efeito estimulante semelhante ao da cafeína (Hammerstone et al., 1999). Cada $100 \mathrm{~g}$ de chocolate contém $5 \mathrm{mg}$ de metilxantina e $160 \mathrm{mg}$ de teobromina, além de $600 \mathrm{mg}$ de feniletilamina (PEA), um estimulante muito parecido com outros produzidos naturalmente pelo organismo, a dopamina e a epinefrina (Lannes, 1997). Durante a última década, pesquisas têm demonstrado que o cacau in natura, alguns produtos de cacau e o chocolate são extraordinariamente ricos num grupo de antioxidantes conhecido como flavonóides, que pertencem a uma ampla e diversa classe de fitoquímicos chamados polifenóis (Freedman et al., 2001).

A quantidade de flavonóides nos produtos de cacau e no chocolate industrializado é dependente da colheita de grãos e condições de processo subseqüentes usado pelos fabricantes de chocolate (Dillinger et al., 2000). Os flavonóides do chocolate são facilmente destruídos pelo calor e inúmeras outras condições comuns ao processo de colheita do cacau e de fabricação do chocolate e, assim, um grande cuidado deve ser tomado pelo fabricante para preservar a existência natural de flavonóides para que quantias significativas permaneçam nos produtos finais (Schmitz, 2001).

Os mecanismos pelos quais os flavonóides presentes no chocolate e no cacau podem melhorar a saúde cardiovascular incluem a redução de danos ao endotélio vascular promovidos pela oxidação do colesterol LDL, assim como a redução da tendência à agregação plaquetária (Wang et al., 2000). A propensão à agregação plaquetária conduz à formação de placas de ateroma responsáveis por infarto do miocárdio, acidente vascular cerebral e gangrenas, embora, os mecanismos de agregação plaquetária exerçam um papel importante na redução de hemorragias durante ferimentos (Rein et al., 2000). Além disso, certos flavonóides do chocolate podem atuar como agentes 
vasoativos, o que reforça o conceito de que os flavonóides presentes no chocolate poderiam promover um fluxo do sangue adequado e um coração saudável (Schmitz, 2001).

\section{Tendências de mercado}

Empresas produtoras de chocolate adquirem favas de cacau de várias partes do mundo e podem prover o líquor de cacau de um único tipo de fava ou misturas de favas diferentes, obtendo blends com sabores específicos (variando grãos e método de torrefação) para agradar o paladar de seus consumidores. Como exemplo de inovação no mercado internacional, pode-se citar a empresa belga Barry Callebout que, além de sua linha de barras de chocolate com sabores diferenciados e sua linha de chocolates dietéticos, recentemente lançou o chocolate sem lactose (substituindo o leite por arroz em pó), o chocolate orgânico e também uma linha enriquecida com inulina e oligofrutose (Chocolate, 2003). Pesquisa recente desenvolveu um chocolate sem sacarose e com propriedades funcionais probióticas, através do enriquecimento do chocolate com bactérias lácteas, que são adicionadas na forma pó em iogurtes (Nebesny et al., 2005).

\section{Produtos de chocolate}

\section{Coberturas de chocolate}

Para a confecção dos produtos de chocolate são utilizadas as coberturas de chocolate em estado sólido (necessitando de derretimento) ou líquido. Chocolate cobertura é o produto homogêneo preparado com massa ou pasta de cacau, manteiga de cacau e açúcares, com composição característica ao tipo de chocolate que se refere: amargo, ao leite ou branco. Existem no mercado coberturas que contêm, em sua formulação, gorduras sucedâneas da manteiga de cacau e que, por isso, não podem ser denominadas de chocolate cobertura (Lannes, 1997).

\section{Produtos moldados}

Existem duas formas distintas para a confecção de produtos de chocolate: recobertos, quando o chocolate é derramado sobre um objeto doce (um núcleo) eliminando todo o excesso por agitação ou sopro; ou moldados, quando o chocolate é colocado em moldes para solidificar, podendo ser recheados ou não (Beckett, 1994).

Os equipamentos utilizados na confecção dos produtos moldados são constituídos basicamente de uma temperadeira, bicos dosadores, esteira vibratória para retiradas de bolhas de ar, túnel de resfriamento e esteira para alimentação das máquinas de embalagem (Minifie, 1983).

O modelo dos moldes é um importante fator para a qualidade do produto final. Moldes com ângulos muito acentuados apresentarão dificuldade na etapa em que irá se retirar o produto. Moldes que possuem muitos detalhes também poderão dificultar a retirada de bolhas de ar do chocolate. Por outro lado, moldes com superfície muito plana poderão propiciar o aparecimento de manchas na superfície do produto. O chocolate muito viscoso, além de prejudicar o preenchimento do molde, não permite a retirada das bolhas de ar (Technology, 1995; Martin, 1994).

Originalmente os moldes eram feitos de metal. Eram considerados pesados e produziam muito ruído durante o processamento, mas apresentavam boa condutividade térmica, problema enfrentado quando os moldes foram substituídos por material plástico. Atualmente os moldes de plástico (policarbonato) permitem redução do consumo de energia, processamento sem ruído e excelente qualidade no produto final (Barry Callebaut, 2000).

$\mathrm{Na}$ fabricação dos bombons moldados recheados, existe a necessidade de uma etapa prévia de formação da chamada "casquinha" de chocolate. Nesta etapa, os moldes são completamente preenchidos de chocolate, vibrados para retirada de bolhas de ar e invertidos para retirada do excesso de chocolate. Os equipamentos mais antigos exigiam que os recheios fossem depositados nas "casquinhas" a temperaturas inferiores ao do ponto de fusão do chocolate (Minifie, 1983).

Atualmente, os equipamentos permitem o depósito do recheio em alta temperatura, pois possuem zonas de resfriamento imediatamente antes e depois do depósito do recheio, e depois do depósito do fundo do bombom, gerando três estágios durante o processamento (Technology,1995). Equipamentos mais recentes produzem bombons recheados em uma única operação, com depósito simultâneo do chocolate e do recheio, seguido de seu resfriamento. Esta operação pode ser descrita como fill-cool-tip-cool (recheieesfrie-despeje-esfrie) (Martin, 1994).

\section{Bombons}

Bombom é o produto constituído por massa de chocolate ou por um núcleo formado de recheio, recoberto por uma camada de chocolate ou glacê. Pode conter outros ingredientes, desde que não descaracterizem o produto, e apresentar formato e consistência variados. (Brasil, 2005-B).

\section{Chocolate Diet}

O chocolate é conhecido por ser um produto rico em gordura e sacarose. Seu valor calórico pode ser diminuído com uma diminuição na concentração de gordura. Entretanto, quando a concentração de gordura é menor que $27 \%$ de seu peso, o chocolate perde a sua suavidade e seu derretimento na boca (Nebesny, Zyzelewicz, 2005). 
Os principais ingredientes do chocolate dietético, junto com os tradicionalmente utilizados, como pasta de cacau, manteiga de cacau, leite em pó etc, são manitol, maltitol, xilitol, frutose e sorbitol (Beckett,1994). Formas cristalinas anidras de polióis como o isomalte, maltitol e lactitol são muito mais fáceis de trabalhar do ponto de vista de processo (Nebesny, Zyzelewicz, 2005).

A alta higroscopicidade dos polióis, com exceção do isomalte, requer cuidados durante o manuseio e processamento do chocolate para prevenir a captura de umidade. $\mathrm{O}$ excesso de umidade pode resultar em um chocolate viscoso, difícil de ser moldado e com um mouthfeel (percepção na boca) desagradável e pegajoso (Zumbé, 2001).

O conteúdo de açúcar no chocolate é em torno de 30 a 55\% (Urbanski, 2003). A substituição da sacarose por um agente de corpo e edulcorante, como o isomalte, permite o desenvolvimento de um chocolate com características físicoquímicas e sensoriais satisfatórias (Nebesny, Zyzelewicz, 2005).

O maltitol também pode substituir a sacarose na formulação do chocolate. É termoestável e tem propriedade de massa similar à sacarose, não existindo necessidade de mudança no processamento. A troca da sacarose por maltitol não significa que o produto pode ser etiquetado como livre de açúcar, devido ao açúcar presente em outros ingredientes, como o açúcar do leite usado na formulação do chocolate (Urbanski, 2003).

Segundo Zumbé (2001), através da combinação dos ingredientes permitidos, segundo o padrão de identidade proposto pela União Européia para chocolate, podem ser desenvolvidos chocolates preenchendo os seguintes requisitos:

- Chocolate reduzido em açúcar: a sacarose adicionada é substituída por poliól ou uma mistura de um poliol com um carboidrato de baixa digestibilidade (polidextrose ou oligofrutose). Para preencher este requisito o açúcar total não deve ser mais que três-quartos do que num alimento similar padrão.

- Chocolate sem açúcar adicionado: toda a sacarose adicionada é substituída como indicada no item acima.

- Chocolate sem açúcar: no chocolate meio amargo toda sacarose adicionada é substituída (como no item acima), mas para o chocolate ao leite e branco, que contém leite, este pode ser substituído por: concentrado protéico de leite (lactose reduzida), mais gordura de leite (por exemplo: $75 \%$ proteína, $12 \%$ lactose e $1 \%$ gordura) ou isolado de proteína de leite (caseínato de sódio + gordura de leite). Devido à dificuldade de desenvolvimento de aroma característico, que normalmente ocorre na fase de conchagem, no processamento do chocolate, pela presença da sacarose e lactose, é necessária a adição de aromas de leite e chocolate para compensação.

- Chocolate com calorias reduzidas: a substituição da sacarose por polióis resulta numa redução calórica de apenas $12 \%$ em média, não atingindo o requisito de redução de 30\% da legislação. Pode-se conseguir essa redução com uma substituição de 50\% de poliól e 50\% de carboidrato de baixa digestibilidade (polidextrose). Essas formulações tendem a ser mais viscosas e ter um mouthfeel mais seco e pegajoso, que pode ser mascarado com a adição de emulsificantes especiais. Avanços têm sido feitos com o uso de tecnologias de processos mais modernos e também pelo uso de substitutos de gordura com menor valor calórico.

O chocolate dietético, em geral, é vendido em barras. Podem-se criar produtos banhados e recheados destinados aos apreciadores de chocolate dietético ou para os diabéticos (Beckett,1994). É recomendado que o tamanho das porções dos produtos não sejam excessivas e limitadas à aproximadamente 60 gramas. $\mathrm{O}$ uso de polidextrose e inulina também é recomendado nas formulações sem açúcar, porque diminui a quantidade de poliól e assim diminui o risco de desconforto gastrintestinal (Zumbé, 2001).

\section{Ingredientes e sua funcionalidade}

\section{Açúcares}

A sacarose é um dissacarídeo formado por uma molécula de glicose em uma molécula de frutose, sendo responsável pelo sabor doce e pelo agente de corpo dos produtos. Muitos produtos de confeitaria utilizam as propriedades especiais de solubilidade e cristalização da sacarose, sozinha ou combinada com outros "açúcares", tais como xarope de glicose (xarope de milho) e açúcar invertido (Charley, Weaver, 1998). Existem basicamente dois grupos de uso: (1) aqueles em que os açúcares estão totalmente em solução (caramelos macios e duros, toffees e geléias) e (2) aqueles em que os açúcares estão parcialmente em soluções e parcialmente na forma de cristais sólidos de açúcar suspensos na solução (fondants, fudge, marshmallows e nougats). Outros ingredientes, como leite e gorduras, podem modificar estes produtos (Minifie,1983).

O açúcar invertido é obtido a partir da hidrólise da sacarose, por meio químico (processo ácido) ou meio enzimático (enzima invertase), sendo constituído por partes iguais de frutose e dextrose. O xarope de glicose é obtido a partir de matérias-primas ricas em amido como batata, mandioca e milho. A conversão do amido em xarope de glicose pode ser obtida por hidrólise ácida, ácida enzimática ou por meio enzimático e o xarope obtido, dependendo do grau de hidrólise e do método empregado, se compõe de diferentes proporções de dextrose, maltose e 
polissacarídeos (Belitz, Grosch, 1987). Os xaropes de glicose são classificados em função do grau de hidrólise do amido, empregando-se o parâmetro DE (valor equivalente de dextrose). Quanto maior o DE, maior o grau de hidrólise sofrido pelo amido e, portanto, maior a proporção de dextrose no xarope (Charley, Weaver, 1998).

O xarope de glicose e o açúcar invertido apresentam algumas propriedades funcionais que são responsáveis pela qualidade dos produtos. Entre estas, destacam-se o poder edulcorante maior do que o da sacarose, a viscosidade, a perfeita solubilidade, a higroscopicidade e o controle da cristalização. Além disso, podem reduzir a atividade de água dos recheios dos bombons, prolongando a vida de prateleira e conferindo boas propriedades de textura e brilho (Jeffery, 1993).

\section{Leite em pó}

Entende-se por leite em pó o produto obtido por desidratação do leite de vaca, desnatado ou parcialmente desnatado e apto para alimentação humana, mediante processo tecnologicamente adequado. A classificação é feita pelo conteúdo de matéria graxa: integral (maior ou igual a 26,0\%); parcialmente desnatado (entre 1,5 a $25,9 \%$ ) e desnatado (menor que 1,5\%) (Engetecno,1996). O leite em pó aumenta o valor nutritivo dos alimentos e influencia na viscosidade e textura, contribuindo, também, para diminuição da umidade e aumento da vida de prateleira (Charley, Weaver, 1998).

\section{Leite condensado}

O leite condensado é feito de leite integral, removendo-se metade da água. Açúcar é adicionado em quantidade suficiente (aproximadamente $44 \%$ ) para preservar o leite, que é então enlatado. O leite não é esterilizado, mas, devido à alta concentração de açúcar, ele se mantém bem, quando utilizado em recheios, aumenta o valor nutritivo e organoléptico e influencia na viscosidade e textura (Charley, Weaver, 1998).

\section{Gorduras substitutas}

Desde os anos 30 existe um interesse maior no uso de gorduras que não a manteiga de cacau na produção de confeitos, devido à incerteza de suprimentos e aos custos da manteiga de cacau, dependentes do mercado flutuante do grão de cacau (Lannes, Gioielli, 1998).

Como aditivos são permitidos a lecitina, lecitina sintética, vanilina e algumas vezes sal. A lei permite até $5 \%$ de manteiga de cacau na receita de outro tipo de gordura vegetal (Brasil, 2005).

Quando uma gordura de composição diferente é adicionada à manteiga de cacau, a forma cristalina da gordu- ra resultante é geralmente alterada, produzindo alteração no perfil de fusão da gordura denominado incompatibilidade (Lannes, 1993).

Para substituí-la de maneira total ou parcial, a indústria e pesquisadores têm trabalhado no desenvolvimento de sucedâneos, produzindo gorduras com características que atendam às exigências dos consumidores. Estes são classificados (Deman, Deman, 1994; Lannes, 1993; Lannes, Gioielli, 1998; Lipp, Anklam, 1998; Timms, 2001) como:

- Substitutos (CBS - "cocoa butter substitutes" e CBR "cocoa butter replacers") - similares à manteiga de cacau em suas propriedades físicas, mas não totalmente compatíveis para misturas. Podem se dividir em láuricas (CBS) e não-láuricas ( $\mathrm{CBR}$ );

- Equivalentes (CBE - "cocoa butter equivalents") - similares em suas propriedades físicas e químicas, sendo compatíveis para misturas em qualquer proporção, pois contêm quase os mesmos ácidos graxos e acilgliceróis da manteiga de cacau.

$\mathrm{O}$ uso de gorduras especiais em recheios cremosos deve ser compatível com a gordura da cobertura, sendo que pode ocorrer migração do recheio para a cobertura ou viceversa. Esse processo pode afetar a integridade e aparência do produto. Recheios com gordura não-láurica apresentam algum grau de compatibilidade com todos os tipos de gorduras de coberturas.

Assim, a gordura representa a fase contínua do recheio, contendo outros ingredientes dispersos, como açúcar, sólidos de cacau e sólidos do leite. Para melhor escolha do sistema de gordura utilizado na elaboração do recheio, alguns critérios devem ser considerados, como ponto de fusão, firmeza, contração, adesividade, tamanho dos cristais, velocidade de solidificação, velocidade de liberação de aromas, estabilidade à temperatura desejada, gordura láurica e não-láurica, miscibilidade com os outros componentes do recheio, habilidade de aeração e estabilidade dos aromas (Vissotto, 1997)

\section{Cacau em pó}

É a parte sólida obtida a partir da prensagem hidráulica da massa de cacau, denominada torta, é moída e resfriada a temperatura controlada. No pó de cacau, os atributos mais importantes são a cor e a finura. A variação de cor é obtida no processo de alcalinização (para aumentar sua solubilidade) e pela finura controlada através de peneiras utilizadas no moinho (Bouzas, Brown, 1999). Sendo um sólido de cacau, nas formulações de recheio ele contribui para textura, cor e sabor e, também, para a redução de atividade da água, aumentando a vida de prateleira. Além disso, a coloração final dos produtos de chocolate desencadeia expectativas de paladar, de forma que o tipo e a quan- 
tidade de pó de cacau empregado podem constituir a diferença entre um produto de sucesso e um produto que fracassa (Oorschot, 2001). O tipo de cacau utilizado e as misturas entre diferentes tipos definem a particularidade para cada tipo de chocolate produzido.

\section{Sorbitol}

É o poliol mais conhecido da família dos álcoois poli-hídricos Sua aplicação na indústria de alimentos é amplamente difundida. Devido à sua capacidade de reter umidade, é muito utilizado como um agente umectante, mantendo o alimento fresco por um período maior de tempo (Deis, 1993). Outras propriedades podem ser citadas: espessante, edulcorante, inibidor de cristalização, plastificante, anticongelante (reduz o ponto de congelamento) e crioprotetor. O sorbitol extraído naturalmente das frutas (ameixa, cereja, maçã, pêssego etc) é economicamente inviável para aplicação em confeitos. Para este fim, é utilizado sob a forma de solução $70 \%$ p/p em água, obtido industrialmente através da hidrogenação catalítica da glicose (Le, Muldering, 2001). De acordo com a legislação vigente, o uso de sorbitol para bombons e similares está limitado ao valor máximo de $5 \%$ sobre o peso total do recheio (Brasil, 2005-B).

\section{Lecitina de soja}

A lecitina de soja é um emulsificante, substância química que pertence ao grupo dos aditivos conhecidos como tensoativos. Possui uma porção hidrofílica, que se liga à água e aos ingredientes solúveis em água, e uma porção lipofílica que possui afinidade com gorduras e ingredientes solúveis neste meio (Hurst, Martin Jr, 1980).

As principais funções dos emulsificantes são: possibilitar uma homogeneização perfeita entre gordura e água; estabilizar a emulsão evitando que haja separação da gordura do produto; proporcionar uma sensação de maior quantidade de gordura no produto; proporcionar plasticidade, suavidade e antiaderência ao produto e distribuir melhor o aroma (Wendel, 2001).

A lecitina de soja é um componente importante no chocolate porque ela reduz of fat bloom (migração de gordura para a superfície), assim como a viscosidade da massa de chocolate e valor de rendimento. O efeito nas características do chocolate depende de sua quantidade e um excesso pode ter um resultado negativo nas propriedades reológicas e sensoriais, podendo deixar um gosto amargo e desagradável em concentrações acima de $0,4 \mathrm{~g} / 100 \mathrm{~g}$ (Nebesny, Zyzelewicz, 2005).

\section{Aromatizantes ou Aromas}

São substâncias ou misturas de substâncias com pro- priedades odoríferas e ou sápidas, capazes de conferir ou intensificar o aroma e ou sabor dos alimentos. Para efeitos do Regulamento Técnico RDC n ${ }^{\circ}$ 2, de 15 de janeiro de 2007, os aromatizantes classificam-se em naturais ou sintéticos (Brasil, 2007).

$\mathrm{Na}$ indústria de chocolates é bastante utilizada a vanilina em pó na produção do chocolate e para recheios aromas líquidos sintéticos são bastante aplicados.

\section{Invertase}

Trata-se de uma glicoproteína que se localiza na parede celular do Saccharomyces cerevisiae, sua principal fonte, onde podemos encontrar duas formas dessa enzima com igual capacidade hidrolítica. É enzima capaz de converter a sacarose em açúcar invertido (partes iguais de glicose e frutose), impedindo ou retardando a cristalização e retendo melhor a umidade. Alguns fatores como temperatura de adição e armazenamento, $\mathrm{pH}$, umidade, proporção de álcool, quantidade adicionada, podem influenciar diretamente na sua ação (Groves, 1981). As melhores condições de atividade são: temperatura menor que $70^{\circ} \mathrm{C}, \mathrm{pH}$ entre 3,8 e 5,2 e quantidade adicionada de 0,1 a $0,2 \%$ nas formulações. É utilizada na forma líquida.

\section{Substitutos de açúcares}

Os desejos dos consumidores mudaram e aumentaram os requisitos para aceitação dos produtos dietéticos. Os produtores perceberam que o sabor e a textura são fundamentais para o sucesso do produto no mercado (Caderno..., 2003). Os produtos elaborados com sacarose geralmente se destacam pela aparência e sabor. Além do sabor doce, a sacarose aumenta a viscosidade do meio, conferindo textura adequada e estabilidade. A utilização de edulcorante pode ser adequada em relação ao sabor, mas, muitas vezes, não proporciona as características de textura e aparência desejada (Oliveira et al., 2004).

Os indivíduos que precisam consumir produtos com substituição da sacarose procuram produtos que sejam dotados de características sensoriais próximas às dos produtos formulados com sacarose (Cardoso et al., 2004). Além do poder dulçor, atualmente os substitutos de açúcares são utilizados como agentes de corpo, textura, realçadores de sabor e propriedades prebióticas (Hirayama, 2002). A única forma de se avaliar a aceitação de um edulcorante é pela análise sensorial. Sua utilização deve considerar a quantidade de ingestão diária aceitável, o tipo de produto e a temperatura que será consumido (Cardoso et al., 2004).

O dulçor de todos os edulcorantes é comparado com o da sacarose. O dulçor relativo é a capacidade que possuem certas substâncias de fornecer intensidades diferentes de 
sabor doce, quando comparadas à sacarose, que é considerada padrão de referência, tendo, portanto, o seu dulçor igual a um (Moretto, 1991).

Os edulcorantes podem ser divididos em duas grandes famílias: os que possuem calorias (nutritivos) e os que não as possuem (não-nutritivos) (Geise, 1993). Geralmente são reconhecidos como seguros (GRAS - Generally Recognized As Safe) e como aditivos de alimentos. Esses termos foram definidos pela ementa para aditivos alimentares de $1^{\circ}$ de janeiro de 1958, cuja lei também nomeou o FDA (Food and Drug Administration) como o órgão que aprova a segurança de todos os aditivos (American Dietetic Association, 1998).

Os limites de segurança são definidos em termos de Ingestão Diária Aceitável - IDA, medida em $\mathrm{mg} / \mathrm{kg}$ de peso corporal. Estes limites são estabelecidos a partir do NOEL (no-effect level), que corresponde à quantia de um determinado aditivo que pode ser ingerida todos os dias por um animal sem causar dano detectável. Essa medida é dividida por um fator de segurança (100) e é a resultante desta operação que será definida como IDA para humanos (Geise, 1993; Cardello et al., 1997).

Os edulcorantes mais conhecidos são:

\section{- Polióis}

São obtidos por hidrogenação da maltose, lactose, palatinose, glicose e xilose. Apresentam valor energético baixo quando comparados com a sacarose, apresentam pouca ou nenhuma ação insulínica porque são absorvidos lentamente ou de forma incompleta pelo intestino e são produtos aprovados ou Generally Recognized as Safe (GRAS) (Tsuneyuki, Nakamura, 2002).

Estão disponíveis em várias formas cristalinas ou como xaropes líquidos. O corpo, mouthfeel (percepção na boca), e sabor podem ser comparados com os da sacarose, mas o grau de doçura é menor, podendo ser elevado com o uso de adoçantes intensos. A solubilidade varia com a temperatura, sendo um fator importante em produtos de confeitaria, onde é necessário o uso de um xarope de poliol combinado aos ingredientes corretos para minimizar o aparecimento de cristais. A higroscopicidade varia com a umidade relativa, a captura de umidade vai limitar a vida de prateleira dos produtos (Zumbé, 2001).

Os polióis e suas principais características (AlmeidaMuradian, Penteado,1990; Geise, 1993; American Dietetic Association, 1998; Le, Muldering, 2001; Zumbé, 2001; Nabors, 2002; Urbanski, 2003) são apresentados a seguir:

Sorbitol - agente umectante e melhorador de textura, poder dulçor 0,6 maior que a sacarina e detentor de ação refrescante. Apresenta estabilidade química e térmica, não participando da reação de Maillard. Muito utilizado em produtos que tendem ao endurecimento e ressecamento como doces, chocolate e recheios. O seu uso em excesso apresenta efeito laxativo (Medeiros, Lannes, 2000).

Isomalte ou isomaltitol (Palatinit $\AA$ ) - é obtido exclusivamente a partir da sacarose, através de um processo patenteado. Além de ser um edulcorante, é um agente de corpo que pode substituir o açúcar na proporção de 1:1. Pode ser combinado com edulcorantes intensos, para se obter o perfil de doçura desejado. Não é metabolizado no intestino delgado e é fermentado principalmente no intestino grosso, explicando seu baixo valor calórico de $2 \mathrm{kcal}$ por grama. A utilização em excesso possui efeito laxativo. Apresenta baixa higroscopicidade, não absorvendo umidade a temperaturas de até $25^{\circ} \mathrm{C}$ e umidades relativas do ar de até $85 \%$, permitindo o desenvolvimento de produtos altamente estáveis à estocagem. Resistente aos ácidos e à hidrólise enzimática. É ideal para ser utilizado em grande variedade de chocolates (ao leite ou amargos) coberturas e recheios, com calorias reduzidas ou para diabéticos, pois tem um sabor comparável ao do açúcar e não apresenta sensação de resfriamento (refrescância) (Zumbé, 2001; Palatinit, 2003; Urbanski, 2003);

Eritritol - pó branco e cristalino, sem odor, com 70\% do poder dulçor da sacarose, pouco solúvel em água, nãohigroscópico. Estável a altas temperaturas e ampla faixa de pH. É utilizado em gomas de mascar, chocolate e confeitos (Zumbé, 2001; Palatinit, 2003; Urbanski, 2003);

Manitol - apresenta baixa solubilidade sendo nãohigroscópico (permitindo sua utilização como agente antiaderente). Amplamente utilizado em gomas de mascar e coberturas de chocolate. O seu uso em excesso apresenta efeito laxativo (Zumbé, 2001; Palatinit, 2003; Urbanski, 2003);

Lactitol - possui baixa higroscopicidade, excelente estabilidade térmica e microbiológica. Confere alta viscosidade às soluções. Ajuda a manter a crocância e aumenta a vida de prateleira de biscoitos e gomas de mascar, podendo ser utilizado também em sorvetes, além de realçar o sabor de chocolate (Zumbé, 2001; Palatinit, 2003; Urbanski, 2003);

Maltitol-não-higroscópico, termoestável, sabor similar à sacarose, de ação refrescante, pode ser utilizado como substituto de gordura e açúcar, como melhorador de cremosidade em chocolates, melhorando suas propriedades organolépticas (Zumbé, 2001; Palatinit, 2003; Urbanski, 2003);

Xilitol - não cariogênico, possui um poder dulçor semelhante à sacarose, e tem ação refrescante, acentuando o sabor da menta em gomas de mascar e confeitos (Zumbé, 2001; Palatinit, 2003; Urbanski, 2003).

Alguns indivíduos podem ser bastante sensíveis mesmo a pequenas doses de polióis, mas para a maioria o consumo de 20 gramas ao dia não deverá causar efeitos 
gastrointestinais indesejáveis e efeito laxativo. Entre os polióis existem diferenças em termos de tolerância. O isomalte (dissacarídeo álcool) é tolerado melhor do que o sorbitol (monossacarídeo álcool) que exerce uma carga osmótica maior no intestino (Zumbé, 2001).

\section{- Edulcorantes Sintéticos}

Edulcorantes intensos não-metabolizáveis que são praticamente nulos em valor energético, mas apresentam intenso poder dulçor, principalmente quando combinados. (Schiffman et al, 1995). Os mais conhecidos são:

Sucralose - é obtida a partir da sacarose, com excepcional estabilidade térmica. É aproximadamente 600 vezes mais doce que a sacarose. Estudos clínicos demonstram que é inócua à saúde, mesmo em níveis de consumo muito superiores ao necessário para adoçar. Pode ser utilizada por diabéticos, fenilcetonúricos e gestantes. Como apresenta alto poder dulçor, há necessidade de correção do "corpo" com outro ingrediente, como a polidextrose, que além do baixo valor energético propicia a manutenção do teor de sólidos. Devido à molécula ser extremamente estável, não existe reação com produtos químicos, ácidos, estabilizantes, aromas e corantes (Grice, Goldsmith, 2000; Urbanski, 2003)

Ciclamato -utilizado em combinação com outros edulcorantes, mascara o sabor amargo e é compatível com a maioria dos ingredientes naturais e artificiais. Facilmente solúvel, é estável em diferentes temperaturas e $\mathrm{pH}$ e possui ótima vida de prateleira (Portmann, Kilcast, 1998).

Aspartame - composto pelos aminoácidos ácido aspártico e fenilalanina. Apresenta valor energético equivalente a $0,4 \mathrm{kcal} / \mathrm{g}$, mas devido à quantidade utilizada pode ser considerado desprezível. A qualidade do sabor doce é muito próxima à da sacarose e pode sofrer hidrólise em altas temperaturas e períodos prolongados de processamento. Relativamente solúvel em água e álcool e insolúvel em óleos e gorduras (Calorie Control Council, 2005).

Sacarina - é utilizada em combinação com outros edulcorantes, pois apresenta forte sabor residual e nota metálica. Apresenta vantagens para indústria por ser termoestável e de baixo custo (American Dietetic Association, 1998).

Acessulfame-K - sabor doce suave e não-residual com emprego em adoçantes de mesas e sobremesas. Estável em temperaturas acima de $200^{\circ} \mathrm{C}$ e ampla faixa de $\mathrm{pH}$, sendo estas as vantagens para as aplicações nas indústrias de alimentos (Peck, 1994).

Segundo a legislação brasileira, a Resolução no 3 de 2 de janeiro de 2001 aprova o regulamento técnico para uso de aditivos edulcorantes, estabelecendo seus limites máxi- mos e mínimos. Esta legislação para alimentos e bebidas em dietas com restrição de açúcar, estabelece os seguintes limites máximos por $100 \mathrm{~g}$ ou $100 \mathrm{~mL}$ de produto pronto para o consumo: esteviosídio $0,06 \mathrm{~g}$; acessulfame-K 0,035 $\mathrm{g}$; aspartame $0,075 \mathrm{~g}$; sacarina $0,03 \mathrm{~g}$; sucralose $0,045 \mathrm{~g}$ e ciclamato 0,03 g (Brasil, 2001).

\section{Agentes de corpo}

Os açúcares são responsáveis por diversas propriedades nos produtos e, quando os retiramos em sua totalidade, alteramos drasticamente todas as características desses produtos. O que se espera de um agente de corpo é que mantenha com a maior similaridade possível as propriedades que o açúcar proporciona ao produto tradicional. O agente de corpo deve ser seguro, estável, de baixa caloria, sem sabor residual, solúvel, contribuir com a coloração e apresentar interações com proteínas e amidos similares às do açúcar (Danisco, 2004).

- Maltodextrinas - é uma mistura de sacarídeos nutritivos (simples e complexos), não doces, formados somente por unidades de D-glicose unidas, principalmente, por ligações a-1,4. É produzida pela hidrólise parcial (ácida ou enzimática) do amido, devendo possuir dextrose equivalente (medida dos açúcares redutores na base seca, fornece a extensão da hidrólise que a maltodextrina possui) inferior a 20. Pode ser encontrada na forma de pó branco ou solução concentrada (Imeson, 1997).

- Polidextrose $\left(\operatorname{Litesse}^{\circledR}\right)$ - resultado da associação de componentes naturais como a glicose, o sorbitol e o ácido cítrico. Possui elevada solubilidade e é extremamente estável dentro de uma ampla faixa de $\mathrm{pH}$, temperatura, condições de processo e estocagem. Incolor, não apresenta sabor residual, sendo compatível com açúcar e edulcorantes de alta potência, permitindo o fácil ajuste do teor de doçura. Possui baixo índice glicêmico: 5-7 comparado à glicose (100). Indicado para consumidores que buscam uma dieta com menos carboidratos, inclusive os diabéticos. Reconhecido em muitos países como uma fibra alimentar solúvel (1 kcal/grama), possui, também, efeito prebiótico devido ao estímulo do crescimento de Lactobacillus e Bifidobacterias com tolerância de 90 gramas por dia (Sandrou, Arvanitoyannis, 2000; Danisco, 2004).

\section{Substitutos de gorduras}

Os substitutos de gorduras ideais devem ser compostos de reconhecida segurança para a saúde e que apresente todas as propriedades funcionais e organolépticas com benefício de redução calórica significativa (Calorie Control Council, 2005). Devido aos múltiplos papéis que as gorduras determi- 
nam nas propriedades dos produtos (estabilidade térmica, emulsificação e aeração, lubrificação, sabor e mouthfeel) é normalmente necessário o uso da combinação de diferentes substitutos de gordura para se obter as propriedades esperadas do produto (McClements, Demetriades, 1998).

Os produtos existentes podem ser classificados em dois grupos, de acordo com seu mecanismo de ação: imitadores ou derivados de gordura. Podem ainda ser classificados com base em sua natureza química, dividindo-se em três grupos: os relacionados aos carboidratos, às proteínas ou às gorduras.

Segundo o mecanismo de ação, os imitadores de gordura são uma combinação de água com lipídios ou não lipídios (carboidratos ou proteínas modificados) com propriedades emulsificantes ou que sejam capazes de formar géis especiais e, assim, normalmente atuam como agentes de corpo. Fazem parte deste grupo: celulose, gomas, inulina, maltodextrina, amidos e amidos modificados, proteínas microparticuladas e proteína concentrada de soro modificado. Os derivados de gordura são compostos nãocalóricos ou de baixo valor calórico, com propriedades semelhantes às dos lipídios, cujas ligações ésteres são modificadas (éteres, ésteres de lipídios com açúcares, com poliglicerol, com polietilenoglicol, ou com ácidos policarboxílicos - análogos de lipídios). Fazem parte deste grupo: Olestra, Caprenina, Sorbestrin, lipídios estruturados e Salatrim (Benefat ${ }^{\circledR}$ ) (Altschul, 1989; Singhal, et al., 1991; Sandrou, Arvanitoyannis, 2000; Calorie Control Council, 2005).

- Inulina - segundo ORAFTI, a inulina da família Raftiline ${ }^{\circledR}$ é $100 \%$ vegetal e natural, extraída da chicória por meio da água quente. Na maioria dos casos, a substituição de gordura por inulina nos alimentos se obtém sem grandes mudanças no processo de produção. Os vários tipos de Raftiline ${ }^{\circledR}$ em pó podem ser incorporados com outros ingredientes ou preparados separadamente com água. Os produtos Raftiline ${ }^{\circledR S T}-\mathrm{Gel}(92 \%$ de inulina e $8 \%$ de glicose, frutose e sacarose) e HP-Gel (100\% de inulina) foram concebidos para produzir um creme estável semelhante à gordura, sem necessidade de agitadores de alta potência. Com a utilização de pequenas quantidades de inulina, se consegue uma melhoria do sabor e da textura dos produtos com baixo teor de gordura. As combinações com polióis ou frutose em chocolates dão excelentes resultados.

- Salatrim (Benefat $\left.{ }^{\circledR}\right)$ - o nome genérico para esta família de gordura reduzida é um acrônimo derivado de Short And Long Acyl Triglyceride Molecule. O Salatrim está licenciado pela Cultor Food Science, mas foi originariamente descoberto pela Nabisco (East Hanover N.J.). Em janeiro de 1996, foi estabelecido o nome Benefat para a família de produtos Salatrim (Kosmark, 1996).

A descoberta e o desenvolvimento inicial do Salatrim foram baseados em dois princípios. Primeiro uma cadeia curta de ácidos graxos, contendo menos de seis carbonos, provendo, assim, menos calorias por unidade de peso do que os ácidos graxos de cadeia longa. Em segundo, ácido esteárico, ácido graxo de cadeia longa que é parcialmente absorvido. $\mathrm{O}$ resultado é uma gordura estruturada com conteúdo de energia reduzida, com 5 kcal/grama (Smith et al., 1994).

As composições do Salatrim são produzidas trocando-se algumas cadeias longas dos ácidos graxos de óleos vegetais hidrogenados como o óleo de canola e óleo de soja, com uma razão específica de ácidos com cadeias curtas como o C-2 acético, C-3 propiônico ou o C-4 butírico. A mistura resultante de triacilgliceróis contém cadeias longas e cadeias curtas de substituintes que estão estrategicamente distribuídas na estrutura do glicerol. O estado físico das composições da família do Salatrim varia de líquido para sólido na temperatura ambiente. Basicamente são dois tipos de mistura: uma delas contendo duas cadeias curtas e uma longa (dishort), a outra sendo formada de duas cadeias longas e uma curta (dilong) (Huang et al., 1994).

Uma avaliação na ingestão de até $30 \mathrm{~g} / \mathrm{dia}$ de Salatrim conclui que não há efeito clínico. Estudos clínicos e de laboratórios mostram não afetar a quantidade de HDL (lipoproteína de alta densidade) ou o LDL (lipoproteína de baixa densidade), não tendo também efeito na absorção de vitaminas lipossolúveis ou micronutrientes (Finley, Leveille,1994).

O Salatrim obteve aprovação do FDA em 1994 como produto GRAS (Generally Recognized as Safe). Na rotulagem de produtos contendo Salatrim, este deverá aparecer nas informações nutricionais como gordura saturada e sua quantidade deverá ser somada à gordura total (Kosmark, 1996). Porém, no Brasil, seu uso ainda não é permitido.

\section{CONCLUSÃO}

O chocolate tem efeito atrativo devido aos ingredientes presentes em sua formulação e aos resultados que estes impõem ao produto final (gordura, açúcar, textura e aroma). Sua digestão se faz facilmente, principalmente devido às gorduras que entram em sua composição: $39 \%$ de ácidos graxos não-saturados, sendo $37 \%$ de ácido oléico e $2 \%$ de ácido linoléico, indispensável ao organismo. A digestibilidade da manteiga de cacau é excelente. Baseando-se nestes ingredientes presentes no chocolate, aqueles que serão utilizados em recheios e em seus produtos deverão seguir o mesmo caminho para que o doce também se torne nutricionalmente interessante. 


\section{ABSTRACT}

\section{Ingredients used in chocolate industry}

Chocolate is a product widely consumed throughout the world that has seen generally increasing production trends over the last years. Making chocolate requires an understanding of how the consumer perceives it. The preferred type of chocolate varies from country to country. The different tastes and uses for chocolate reflect the histories of the industry in different places. The taste of chocolate is partially determined by the chemistry of the product. Taste depends on the release offlavor compounds in the mouth and nose, while perceived texture is a function of the way in which the material melts and breaks up in the mouth. Most chocolates that are available on the market throughout the world are made from very similar ingredients, yet have very different flavors. Some manufacturers have specific house flavors, and very often this is due to specific changes during the manufacturing process, where changes in holding times, acidity and temperature can give rise to very different flavors in the final chocolate. For this reason, chocolate manufacturing conditions are often kept a closely guarded secret. The ingredients used in chocolate and confectionery products have an important role in consumers acceptance and presentation of the product.

UNITERMS: Chocolate. Confectionery. Ingredients/food industry. Food Industry.

\section{REFERÊNCIAS BIBLIOGRÁFICAS}

ALMEIDA-MURADIAN, B.L.; PENTEADO, M.V.C. Edulcorantes em alimentos: uma revisão. Bol. Soc. Bras. Ciênc. Tecnol. Aliment., Campinas, v.24, n.1/2, p.1-11, 1990.

ALTSCHUL, A.M. Low calorie foods. Food Technol., Chicago, v.43, n.4, p.113-125, 1989.

AMERICAN DIETETIC ASSOCIATION. Use of nutritive and nonnutritive sweeteners: position of American Dietetic Association. J. Am. Diet. Assoc., Orlando, v.98, p.580$587,1998$.

BARRY CALLEBAUT. Chocolate for the professional. Food Manufactures Product \& Applications. Possible Applications for our Products . Disponível em: $<\mathrm{http}: / /$ w w w . barry - c a lle ba u t c o m/ma in/ frameset.asp?reference $=01 \% 2 \mathrm{D} 01 \& \mathrm{lang}=$ en $\&$ sess $=505559279 \&>$. Acesso em: 15 ago. 2004 .
BECKETT, S. T., (Ed.). Fabricación y utilización industrial del chocolate. Zaragoza: Acribia, 1994. p.276-277.

BELITZ, H. D.; GROSCH, W. Food chemistry. Berlin, New York: Springer Verlag, 1987. p.128-200.

BONZAS, J.; BROWN, B. D. Interactions affecting microstructure, texture, and rheology of chocolate confectionery products. In: GAONKAR, A.G., (Ed.). Ingredient interactions: effects on food quality. New York: Marcel Dekker, 1999. p.451-523. (Food Science and Technology, 66).

BRASIL. Ministério da Saúde. Agência Nacional de Vigilância Sanitária. Legislação. VisaLegis. Resolução RDC n.264, de 22 de setembro de 2005. Aprova Regulamento Técnico para Fixação de Identidade e Qualidade de Chocolate e Chocolate Branco. Disponível em: <http://e-legis.anvisa.gov.br/leisref/public/ showAct.php?id $=18823 \&$ word $=$ chocolate $>$. Acesso em: 10 nov. 2006.(A)

BRASIL. Ministério da Saúde. Agência Nacional de Vigilância Sanitária. Legislação. VisaLegis. Resolução RDC n.265, de 22 de setembro de 2005. Aprova Regulamento Técnico para balas, bombons e gomas de mascar. Disponível em: <http://e-legis.anvisa.gov.br/ leisref/public/showAct.php?id=18824>. Acesso em: 10 nov. 2006. (B)

BRASIL. Ministério da Saúde. Agência Nacional de Vigilância Sanitária. Legislação. VisaLegis. Resolução RDC n.3, de 2 de janeiro de 2001. Aprova o "Regulamento Técnico que aprova o uso de Aditivos Edulcorantes, Estabelecendo seus Limites Máximos para os Alimentos". Disponível em: <http://e-legis.bvs.br/ leisref/public/showAct.php?id=3181\&word $>$. Acesso em: 15 set. 2005.

BRASIL. Ministério da Saúde. Agência Nacional de Vigilância Sanitária. Legislação. VisaLegis. Resolução $R D C n .2$, de 15 de janeiro de 2007. Aprova a "Resolução da Diretoria Colegiada que aprova o regulamento técnico sobre aditivos aromatizantes, que consta como anexo da presente Resolução". Disponível em: < http:// www.anvisa.gov.br/legis/resol $/ 2007 / \mathrm{rdc} /$ 02_170107rdc.htm>. Acesso em: 09 jul. 2007.

CADERNO de pesquisas \& inovações: Guia 2003. Food Ingredientes, São Paulo, v.4, n.24, p.105-118, 2003. 
CALORIE CONTROL COUNCIL. Fat Replacers: food ingredients for healthy eating. Disponível em: $<$ http:// www.caloriecontrol.org/fatrepl.html $>$. Acesso em: 05 maio 2005.

CALORIE CONTROL COUNCIL. Low Calorie Sweeteners. Aspartame. Disponível em: $<$ http:// www.caloriecontrol.org/aspartame.html $>$. Acesso em: 24 ago. 2005

CARDELLO, H. M. A. B., DANÁSIO, M. H. Edulcorantes e suas características: revisão. Bol. Soc. Bras. Ciênc. Tecnol. Aliment., Campinas, v.31, n.2, p.241-247, 1997.

CARDOSO, J. M. P.; BATTACHIO, J. R.; CARDELLO, H. M. A. B. Equivalência de dulçor e poder edulcorante de edulcorantes em função da temperatura de consumo em bebidas preparadas com chá-mate em pó solúvel. Ciênc. Tecnol. Aliment., Campinas, v.24, n.3, p.448-452, 2004.

CHARLEY, H.; WEAVER, C. Sugar, alternative sweeteners and confections. In: .Foods: a scientific approach. Upper Saddle River: Merrill Prentice Hall, 1998. cap.8, p.119-135.

CHARLEY, H.; WEAVER, C. Milk. In: .Foods: a scientific approach. Upper Saddle River: Merrill Prentice Hall, 1998. cap.19, p.308-323.

CHOCOLATE ingredients: bringing chocolate to the whole world. Confect. Prod., Surbiton, v.6, p.8-10, 2003.

DANISCO. Litesse ${ }^{\circledR}$ polidextrose: contribuindo para uma vida saudável. S.1.: DANISCO Sweeteners, 2004. 6p. [Especificação Técnica].

DEMAN, J. M.; DEMAN, L. Specialty fats based on palm oil and palm kernel oil. Malaysian: Malaysian Palm Oil Promotion Council, 1994. 16p. (Palm oil information series).

DEIS, R. Low-calorie and bulking agents. Food Technol., Chicago, v.47, n.12, p.94, 1993.

DILLINGER, T. L.; BARRIGA, P.; ESCARCEGA, S.; JIMENEZ, M.; LOWE, D. S.; GRIVETTI, L. E. Food of the gods: cure for humanity? a cultural history of the medicinal and ritual use of chocolate. J. Nutr., Bethesda, v.130, p.2057S-2072S, 2000.
ENGETECNO ONLINE. Legislação. Leite e Derivados. Leite em Pó. Portaria n.146, de 07 de março de 1996. Regulamento técnico de identidade e qualidade de leite em pó. Disponível em: <http://www.engetecno.com.br/ legislacao/leite_rtfiq_leite_po.htm $>$. Acesso em:05 maio 2005.

FINLEY, J. W.; LEVEILLE, G. A. Clinical assessment of salatrim, a reduced-calorie tricylglycerol. J. Agric. Food Chem., Columbus, v.42, p.581-596, 1994.

FREEDMAN, J.E.; PARKER, C.; LIQING, L.; PERLMAN, J. A.; FREI, B.; IVANOV, V.; DEAK, L. R.; IAFRATI, M. D.; FOLTS, J. D. Select flavonoids and whole juice from purple grapes inhibit platelet. Circulation, Hagerstown, v.103, p.2792-2798, 2001.

GEISE, J.H. Alternative sweeteners and bulking agents. Food Technol., Chicago, v.47, n.1, p.114-126, 1993.

GRICE, H. C.; GOLDSMITH, L. A. Sucralose an overview of the toxicity data. Food Chem. Toxicol., Amsterdam, v.38, suppl.2, p.S1-S6, 2000.

GROVES, R. Ingredients: use them - do not abuse them. Candy Snack Ind., New York, v.146, n.4, p.42, 44, 46, 1981.

HAMMERSTONE, J. F.; LAZARUS, S. A.; MICHELL, A. E.; RUCKER, R. B.; SCHMITZ, H. H. Indentification of Procyanidins in Cocoa (Theobroma Cacao) ad chocolate using high-performance liquid chromatography/mass spectrometry. J. Agric. Food Chem., Columbus, v.47, p.490-496, 1999.

HIRAYAMA, M. Novel physiological functions of oligosaccharides. Pure Appl. Chem., Research Triangle Park, v.74, n.7, p.1271-1279, 2002.

HUANG, A. S.; DELANO, G. M.; PIDEL, A.; JANES, L. E.; SOFTLY, B. J.; TEMPLEMAN, G. J. Characterization of triaylglycerols in saturated lipid mixtures with application to salatrim 23CA. J. Agric. Food Chem., Columbus, v.42, p.453-460, 1994.

HURST, W. J.; MARTIN JUNIOR, R. A. The HPLC separation and quantitation of lecithin in chocolate. J.Am. Oil Chem. Soc., Champaign, v.57, n.9, p.307-310, 1980. 
IMESON, A., ed. Thickening and gelling agents for food. 2.ed. London, New York: Blackie, 1997. 336p.

JEFFERY, M. S. Key functional properties of sucrose in chocolate and sugar confectionery. Food Technol., Chicago, v.47, n.1, p.141-144, 1993.

KOSMARK, R. Salatrim: properties and applications. Food Technol., Chicago, v.50, n.4, p.98-101, 1996.

LANNES, S. C. S. Estudo comparativo entre manteiga de cacau e seus sucedâneos comerciais. São Paulo, 1993. 101p. [Dissertação de Mestrado, Faculdade de Ciências Farmacêuticas, Universidade de São Paulo].

LANNES, S. C. S. Estudo das propriedades fisico-químicas e de textura de chocolates. São Paulo, 1997. 175p. [Tese de Doutorado, Faculdade de Ciências Farmacêuticas, Universidade de São Paulo].

LANNES, S. C. S.; GIOIELLI, L. A. Uso de gorduras vegetais hidrogenadas na indústria de chocolates. Óleos Grãos. São Caetano do Sul, v.8, p.44-46, 1998.

LE, A. S.; MULDERING, K. B. Sorbitol and mannitol. In: NABORS, L. O. B.; GELARDI, R. C., eds. Alternative sweeteners. 3.ed. New York: Marcel Dekker, 2001. p.3441. (Food science and technology, 112).

LIPP, M.; ANKLAM, E. Review of cocoa butter and alternatives fats for use in chocolate. Part A. Compositional data. Food Chem., Amsterdam, v.62, n.1, p.73-97, 1998.

MARTIN, A. V. Chocolate confectionery. In: MAN, C. M. D.; JONES, A. A., (Eds.). Shelf life evaluation of foods. London, New York: Blackie Academic, 1994. p.216-234.

MARTIN JUNIOR, R. A. Chocolate. Adv. Food Res., New York, v.31, p.211-342, 1987.

McCLEMENTS, J.; DEMETRIEDES, K. An integrated approch to the development of reduced-fat food emulsions. Crit. Rev. Food Sci. Nutr., Philadelphia, v.38, n.6, p.511536, 1998.

McGAUlEY, S. E.; MARANGONI, A. G. Static Crystallization behavior of cocoa butter and its relationship to network microstructure. In: MARANGONI A.G.; NARINE, S.S., eds. Physical properties of lipids. New York: Marcel Dekker, 2002. cap.4, p.85-123.
MEDEIROS, M. L.; LANNES, S. C. S. Avaliação da textura de bombons com recheio de longa duração. Rev. Bras. Ciênc. Farm., São Paulo, v.36, supl.1, p.48, 2000. (V Semana de Ciência e Tecnologia da FCF-USP: Resumos).

MINIFIE, B. W. Chocolate, cocoa and confectionery: science and technology. 2.ed. Westport: AVI, 1983. 735p.

MORETTO, L. D. Química do sabor doce. In: UNIVERSIDADE DE SÃO PAULO. Faculdade de Ciência Farmacêuticas. Departamento de Tecnologia Bioquímico-Farmacêutica. Tecnologia de alimentos dietéticos: módulo I: edulcorantes. São Paulo: Departamento de Tecnologia Bioquímico-Farmacêutica, 1991. cap.3, p.38-55

NABORS, L. O. B. Sweet choices: sugar replacements for foods and beverages. Food Techonol., Chicago, v.56, n.7, p.28-34, 2002.

NEBESNY, E.; ZYZELEWICZ, D. Effect of lecithin concentration on properties of sucrose-free chocolate masses sweetened with isomalt. Eur. Food Res. Technol., Berlin, v.220, p.131-135, 2005.

NEBESNY, E.; ZYZELEWICZ, D.; MOTYL, I.; LIBUDZISY, Z. Properties of sucrose-free chocolates enriched with lactic acid bacteria. Eur. Food Res. Technol., Berlin, v.220, p.358-162, 2005.

OLIVEIRA, A. P. V.; FRASSON, K.; ALMEIDA, T. C. A.; BENASSI, M. T. Aceitação de sobremesas lácteas dietéticas e formuladas com açúcar: teste afetivo e mapa de preferência interno. Ciênc. Tecnol. Aliment., Campinas, v.24, n.4, p.627-633, 2004.

OORSCHOT, N. V. Perfeição em matéria de sobremesas e leites achocolatados. Leite Deriv., São Paulo, n.58, p.2831,2001 .

ORAFTI. Inovar com Raftiline ${ }^{\circledR}$. S.1.: s.n., s.d. 8p. [Informativo Técnico].

PALATINIT. Isomalt: dados, fatos e vantagens. Barueri, 2003. 11p. [Especificação Técnica].

PECK, A. Use of acesulfame $\mathrm{K}$ in light and sugar-free baked goods. Cereal Foods World, Saint Paul, v.39, n.10, p.743745, 1994. 
PORTMANN, M. O.; KILCAST, D. Descriptive profiles of synergistic mixtures of bulk and intense sweeteners. Food Qual. Prefer., Kidlington, v.9, n.4, p.221-229, 1998.

REIN, D. S.; HOLT, R. R.; KEEN, C. L.; SCHMITZ, H. H.; FRAGA, C. G. Epicatechin in human plasma: in vivo determination and effect of chocolate consumption on plasma oxidation status. J. Nutr., Bethesda, v.130, p.21095S-2114S, 2000.

SANDROU, D. K.; ARVANITOYANNIS, I. S. Low-fat/ calorie foods: current state and perpectives. Crit. Rev. Food Sci. Nutr., Philadelphia, v.40, n.5, p.427-447, 2000. [Review].

SCHENK, H.; PESCHAR, R. Understanding the structure of chocolate. Radiat. Phys. Chem., Amsterdam, v.71, n.3/4, p.829-835, 2004.

SCHIFFMAN, S. S.; BOOTH, B. G.; CARR, B. T.; LOSEE, M. L.; SATTELY-MILLER, E. A. A.; GRAHAM, B. G. Investigation of synergism: in binary mixtures of sweeteners. Brain Res. Bull., Orlando, v.38, n.2, p.105120, 1995.

SCHMITZ, H. H. Chocolate, flavonoids and heart health. Manuf. Confect., Glen Rock, v.81, n.9, p.95-99, 2001.

SINGHAL, R. S.; GUPTA, A. K.; KULKARNI, P. R. Lowcalorie fat substitutes. Trends Food Sci. Technol., Amsterdam, v.2, n.10, p.241-244, 1991.

SMITH, R. E.; FINLEY, J. W.; LEVEILLE, G. A. Overview of salatrim, a family of low-calorie fats. J. Agric. Food. Chem., Columbus, v.42, p.432-434, 1994.

STAPLEY, A. G. F.; TEWKESBURY, H.; FRYER, P. J. The effects of shear and temperature history on the crystallization of chocolate. J. Am. Oil Chem. Soc., Champaign, v.76, n.6, p.677-685, 1999.
TECHNOLOGY of chocolate molding. Ind. Aliment., Pinerolo, v.34, n.336, p.415-419, 1995. [Editorial Material].

TIMMS, R. E. Chocolate fats. Food Ingredients Anal. Int., Uxbridge, v.23, n.4, p.15-19, 2001.

TSUNEYUKI, O.; NAKAMURA, S. Digestion, absorption, fermentation, and metabolism of functional sugar substitutes and their available energy. Pure Appl. Chem., London, v.74, p.7.1253-1261, 2002.

URBANSKI, J. J. Sugarfree chocolate coatings. Manuf. Confect., Glen Rock, v.83, n.6, p.61-67, 2003.

VISSOTTO, F. Z. Gorduras especiais: aplicação em recheios. Chocotec informativo, Campinas, v.3, n.4, p.3, 1997.

WANG, J. F.; SCHRAMM, D. D.; HOLT, R. R.; ENSUNSA, J. L.; FRAGA, C. C.; SCHMITZ, H. H.; KEEN, C. L. A dose-response effect from chocolate consumption on plasma epicatechin and oxidative damage. J. Nutr., Bethesda, v.130, p.2115S-2119S, 2000.

WENDEL, A. Lecithin in chocolate: the historical start. INFORM, Champaign, v.12, n.8, p.821-823, 2001.

ZUMBÉ,A.; LEE, A.; STOREY, D. Polyols in confectionery: the route to sugar-free, reduced sugar and reduced calorie confectionery. Br. J. Nutr., Wallingford, v.85, suppl.1, p.S31-S45, 2001.

Recebido para publicação em 10 de novembro de 2006. Aceito para publicação em 12 de julho de 2007. 